\title{
Novel approach to rectal foreign body retrieval: A Case Report
}

\section{Tzu-Yi Chuang, Paul Tingey, Hassan Malik, Havish Srinath}

\section{ABSTRACT}

Retained rectal foreign bodies (RFB) are becoming an increasingly common presentation to Emergency Departments, and pose a significant risk of morbidity for patients. If non-operative removal of these objects is unsuccessful, laparotomies or laparoscopies may need to be performed. In this case report, a 43-year-old male presented to hospital requesting assistance removing a RFB. The retained rectal foreign body in question was a 7 cm diameter firm rubber ball. After numerous failed approaches, the RFB was removed using a Kiwi suction device with assistance from our obstetrics and gynaecology colleagues. This paper describes our experiences with this case, and discusses similar events documented in the literature.

Keywords: Rectum, Foreign, Retained, Extraction, Suction

\section{How to cite this article}

Chuang T, Tingey P, Malik H, Srinath H. Novel approach to rectal foreign body retrieval: A Case Report. Int J Case Rep Images 2018;9:100926Zo1TC2018.

Tzu-Yi Chuang ${ }^{1}$, Paul Tingey ${ }^{2}$, Hassan Malik ${ }^{3}$, Havish Srinath $^{3}$

Affiliations: ${ }^{1}$ Surgical Principle House Officer, Department of General Surgery, Ipswich Hospital, Ipswich, Queensland, Australia; ${ }^{2}$ Surgical Intern, Department of General Surgery, Ipswich Hospital, Ipswich, Queensland, Australia; ${ }^{3}$ Surgical Registrar, Department of General Surgery, Ipswich Hospital, Ipswich, Queensland, Australia.

Corresponding Author: Tzu-Yi Chuang, 7/141 Station Road, Sunnybank Queensland, Australia 4109; Email: Thomas0227@hotmail.com

Received: 21 April 2018

Accepted: 22 May 2018

Published: 22 June 2018
Article ID: 100926Zo1TC2018

$* * * * * * * * *$

doi: 10.5348/100926Zo1TC2018CR

\section{INTRODUCTION}

Since the first reported case of Retained Rectal Foreign Bodies (RFBs) in the $16^{\text {th }}$ century, they have become an increasingly common presentation to emergency departments particularly in the last few decades [1-3].

Surgical intervention is often required when a patient presents to hospital with a RFB [3]. Although a few papers have attempted to describe therapeutic protocols for management of colorectal foreign bodies, it is difficult to apply a standard approach as variation in types, shapes and materials of foreign bodies that have been reported across the literature [4-6].

Findings from case reports discourage attempts at transanal extraction in the emergency department as these were found to be associated with a greater than $60 \%$ chance of pushing the foreign body higher into the rectosigmoid region [4-6]. As such, examination under anaesthesia is recommended.

The current methods for retrieval of RFBs include manual extraction, endoscopic extraction, inflated Foley catheter to pull object down, flexible sigmoidoscopy with polypectomy snares or grasped with biopsy forceps, TAMIS extraction, laparoscopic or open advancement with transanal extraction, and laparoscopic or open transmural extraction [7]. Prior to any of these techniques, it is of course essential to rule out and evaluate whether there is perforation subsequent to the RFB. This will determine whether the patient requires urgent laparoscopy or laparotomy without attempting to manually retrieve the RFB transanally.

The Kiwi Cup suction is a single-use, manually operated vacuum designed to make head rotation easier during assisted vaginal deliveries. This instrument was originally approved for distribution following clinical evaluation in 2002 [8]. We here described a novel approach for RFBs retrieval by using the kiwi vacuum suction to remove the foreign body. 


\section{EDORIUM Journals}

\section{CASE REPORT}

A 43-year-old heterosexual male presented to the emergency department requesting assistance removing a retained rectal foreign body. The object in question was a $7 \mathrm{~cm}$ diameter firm rubber ball, placed within his rectum by a female sexual partner 10 hours prior. The patient reported that a second, similar object had been inserted at the same time, but the patient had managed to pass that object prior to seeking medical assistance. The patient had unsuccessfully attempted to remove the foreign body with a spoon, and had caused some rectal bleeding.

Upon presenting to the emergency department, the patient denied any abdominal pain, and reported no ongoing rectal bleeding. All vitals were within normal limits. A hard spherical mass was noted on deep palpation of the suprapubic region.

The following X-rays revealed a thick-walled, spherical foreign body within the rectum as shown in Figure 1 (A and $\mathrm{B}$ ).

On rectal examination, the patient was found to have reduced anal tone, easily admitting two fingers. The patient reported that he had pre-existing flatus incontinence as a result of previous experiences with rectal foreign bodies. The foreign body was palpable in the mid-rectum. With combined suprapubic pressure and digital exploration, the foreign body could not be retrieved in the emergency department. Further attempts to retrieve the object using wall-mounted suction also failed. The patient was consented for an exploration under anaesthetic with possible laparotomy and bowel resection in order to remove this foreign body.

While in theatre, in lithotomy position, numerous strategies were employed in order to remove the foreign body. Given the patient's pre-existing anal sphincter laxity, visualisation of the foreign body was achieved using a large gynaecologic speculum. Application of abdominal pressure was used to milk the object distally. Attempts to remove the foreign body with sponge forceps were unsuccessful. Passing a Foley's catheter proximal to the foreign body, subsequent inflation of the balloon and traction was unsuccessful. The same approach using a larger rectal tube was also unsuccessful. Attempts using small delivery forceps were unsuccessful due to size limitations preventing the delivery forceps from being applied around the foreign body. Wall-mounted suction was attempted intra-operatively with no success. Discussion regarding potential deflation of the ball using a large needle and syringe was deemed too high risk with potential for damage to the rectal wall.

Discussion resulted in the suggestion of contacting the obstetrics and gynaecology team to attempt to apply a Kiwi Cup suction device to the round foreign body, similar to it's application on the head of a baby in an obstructed labour. The obstetrics and gynaecology registrar arrived to theatre, applied the suction device, and successfully removed the ball as shown in Figure 2(A and B). Following foreign body removal, rigid sigmoidoscopy was used to visualize the rectum. Erythema was noted, however there were no perforations. The patient made an uneventful recovery and was discharged from hospital the following day.

\section{DISCUSSION}

Increasing trends of RFBs in patients presenting to hospital have been noted [2]. The diagnosis and management dilemma of RFBs could be sometimes difficult as patients may not disclose the information when they first present to hospital [1-3]. These patients usually present with anorectal, pelvic or lower abdominal pain [2] and approximately $80 \%$ of RFBs are introduced for sexual stimulation [7].

The diagnosis can be made by digital rectal examination, proctoscopy, and plain radiography [1]. Radiographs help to detect the presence of pneumoperitoneum, along with the size, shape and location of the RFBs. Imaging therefore may assist clinicians to determine which extraction approach would be more appropriate as some RFBs are too high of a risk to be removed via the trans-anal approach. Location of the RFBs is also important to identify as it is noted that foreign bodies within the sigmoid colon are 2.5 times more likely to require surgery compared to those in the

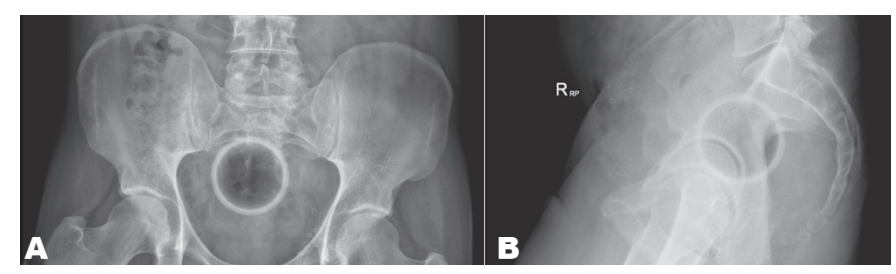

Figures 1 (A and B): Pelvic X-rays displaying $7 \mathrm{~cm}$ diameter foreign body in patient's rectum. Surgical clips are noted overlying the left side of the pelvis due to prior hernia repair.

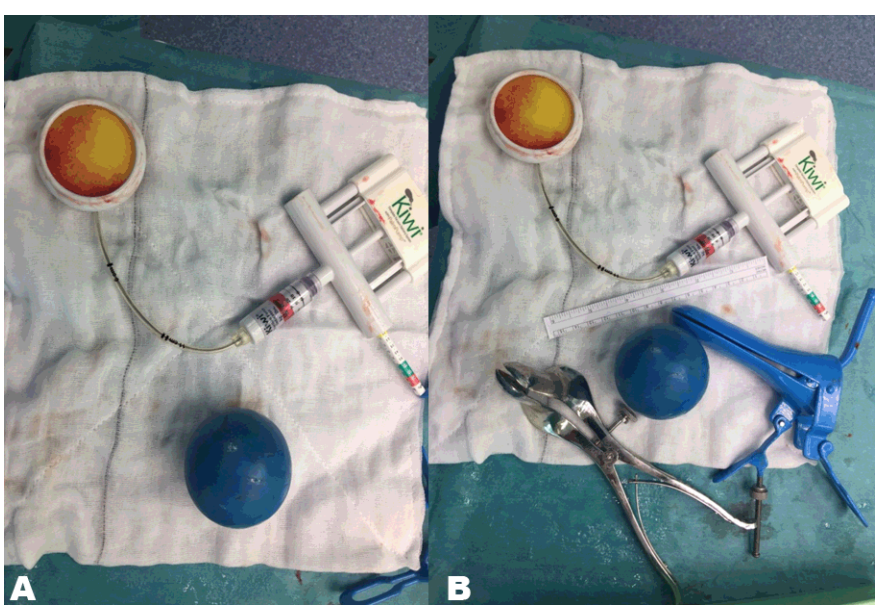

Figure 2(A and B): Rectal foreign body (the blue ball), with instruments used to retrieve it. 


\section{EDORIUM Journals}

rectum [3]. Some authors also suggest performing a CT abdomen/pelvis if RFBs are in situ for greater than 24 hours [4-6].

Use of an obstetric vacuum extractor for nongynaecological procedures have been previously described [9-11]. The first case was reported in 1996 by Johnson et al. [10] with successful extraction of a glass bottle using obstetric vacuum. Feigelson et al. [9] suggested that softcup instruments with a manual vacuum pump is a useful technique for removal of large spherical objects. The vacuum cup provides the advantage of conforming to the objects surface easily without causing significant trauma to surrounding tissue.

Post RFB retrieval observation is recommended by Coskun et al. [3]. Post-extraction endoscopy, serial abdomen examination, and plain radiographs are required prior to discharge. Traumatic disruption of the anal sphincter is an important discussion with patients as it can vary from mild to severe faecal incontinence depending on the degree of injury [7]. Any sphincter repair surgery should be delayed and evaluated carefully with imaging tests and clinical symptoms [3]. In our case, we conducted rigid sigmoidoscopy to confirm no perforation occurred subsequent to the retrieval of the RFB. As this patient already had pre-existing anal incontinence, his persistent anal incontinence following the procedure was not attributed to the removal technique. He was referred to the colorectal specialty team for ongoing management after discharge from the hospital. By avoiding various potential complications from intra-abdominal laparoscopy or laparotomy, patient's morbidity is believed to have been significantly reduced. The authors of this paper would highly recommend utilization of gynaecology instruments in the future for any large, spherical rectal foreign bodies as a first option.

\section{CONCLUSION}

The application of the Kiwi Cup suction device was highly successful and prevented this patient from requiring a laparotomy.

\section{REFERENCES}

1. Cawich SO, Thomas DA, Mohammed F, Bobb NJ, Williams D, Naraynsingh V. A management algorithm for retained rectal foreign bodies. Am J Mens Health 2017 May;11(3):684-92.

2. Ayantunde AA, Unluer Z. Increasing trend in retained rectal foreign bodies. World J Gastrointest Surg 2016 Oct 27;8(10):679-84.

3. Coskun A, Erkan N, Yakan S, Yildirim M, Cengiz F. Management of rectal foreign bodies. World J Emerg Surg 2013 Mar 13;8(1):11.

4. Kasotakis G, Roedigerb L, Mittalc S. Rectal foreign bodies: A case report and review of the literature. International Journal of Surgery Case Reports 2012;3(3):111-15.
5. Lake JP, Essani R, Petrone P, Kaiser AM, Asensio J, Beart RW Jr. Management of retained colorectal foreign bodies: Predictors of operative intervention. Dis Colon Rectum 2004 Oct;47(10):1694-8.

6. Rodríguez-Hermosa JI, Codina-Cazador A, Ruiz B, Sirvent JM, Roig J, Farrés R. Management of foreign bodies in the rectum. Colorectal Dis 2007 Jul;9(6):543-8.

7. Cologne KG, Ault GT. Rectal foreign bodies: What is the current standard? Clin Colon Rectal Surg 2012 Dec;25(4):214-8.

8. Hayman R, Gilby J, Arulkumaran S. Clinical evaluation of a "hand pump" vacuum delivery device. Obstet Gynecol 2002 Dec;100(6):1190-5.

9. Feigelson S, Maun D, Silverberg D, Menes T. Removal of a large spherical foreign object from the rectum using an obstetric vacuum device: A case report. Am Surg 2007 Mar;73(3):304-6.

10. Johnson SO, Hartranft TH. Nonsurgical removal of a rectal foreign body using a vacuum extractor: Report of a case. Dis Colon Rectum 1996 Aug;39(8):935-7.

11. Bak Y, Merriam M, Neff M, Berg DA. Novel approach to rectal foreign body extraction. JSLS $2013 \mathrm{Apr}-$ Jun;17(2):342-5.

\section{$* * * * * * * * *$}

\section{Acknowledgements}

We would like to thank our obstetrics and gynaecology colleagues for their valuable contributions in this case.

\section{Author Contributions}

Tzu-Yi Chuang - Acquisition of data, Analysis and interpretation of data, Drafting the article, Revising it critically for important intellectual content, Final approval of the version to be published

Paul Tingey - Analysis and interpretation of data, Drafting the article, Revising it critically for important intellectual content, Final approval of the version to be published

Malik Hassan - Acquisition of data, Revising it critically for important intellectual content, Final approval of the version to be published

Havish Srinath - Acquisition of data, Revising it critically for important intellectual content, Final approval of the version to be published

\section{Guarantor of Submission}

The corresponding author is the guarantor of submission.

\section{Source of Support}

None

\section{Consent Statement}

Written informed consent was obtained from the patient for publication of this case report.

\section{Conflict of Interest}

Authors declare no conflict of interest. 


\section{EDORiUM Journals}

\section{Copyright}

(C) $2018 \mathrm{Tzu}-\mathrm{Yi}$ Chuang et al. This article is distributed under the terms of Creative Commons Attribution License which permits unrestricted use, distribution and reproduction in any medium provided the original author(s) and original publisher are properly credited. Please see the copyright policy on the journal website for more information.
Access full text article on other devices

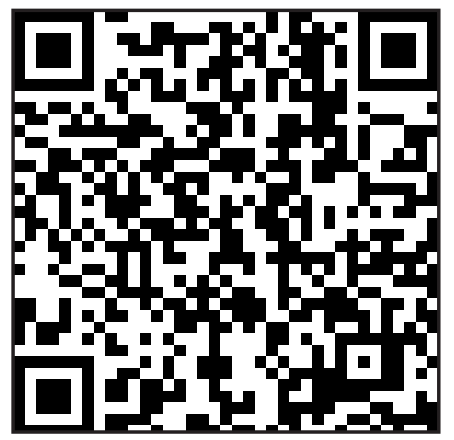

Access PDF of article on other devices

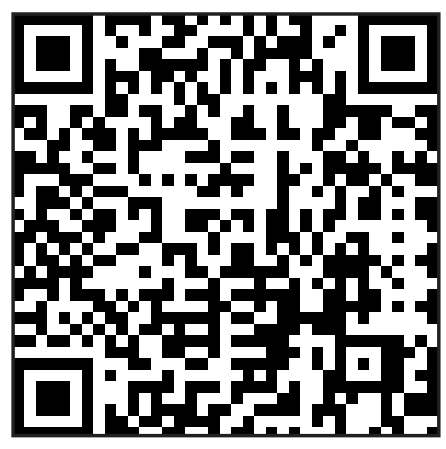

